\title{
Effect of thiazide on rates of bone mineral loss: a longitudinal study
}

\author{
Richard Wasnich, James Davis, Philip Ross, John Vogel
}

\begin{abstract}
Objective-To determine the effect of thiazide diuretic drugs on rates of bone mineral loss.

Design-Longitudinal, observational study with a mean follow up of five years.

Setting-Hawaii Osteoporosis Center, Honolulu.

Subjects-1017 Japanese-American men born between 1900 and 1920, of whom 378 were treated for hypertension (study group) and 639 did not have hypertension (control group).
\end{abstract}

Intervention-Thiazide diuretics were taken by 325 men for a mean of 11.9 years; 53 men took antihypertensive drugs other than thiazides.

Main outcome measure-Rate of bone loss estimated from serial photon absorptiometric scanning at three skeletal sites (calcaneus, distal radius, and proximal radius).

Results-Rates of bone loss at all three sites were significantly reduced among thiazide users when compared with controls. The reductions in loss rate ranged from $28.8 \%(p=0.02)$ (distal radius) to $49.2 \%$ $(\mathbf{p}=\mathbf{0 . 0 0 0 5})$ (calcaneus) relative to the controls. At all three sites the men taking other antihypertensive drugs had faster loss rates $(22 \cdot 6-43 \cdot 1 \%)$ than those of the controls but the difference was significant only for the distal radius.

Conclusion-Thiazide diuretics slow the rate of bone loss in elderly men.

\section{Introduction}

The thiazide diuretics have diverse effects on electrolyte and mineral metabolism, including reduced urinary calcium excretion and increased urinary excretion of sodium, phosphate, and magnesium.' Previous cross sectional studies of both men and women have found greater bone mass and a reduced prevalence of fracture among patients taking thiazides. ${ }^{2-8}$ Two case-control studies have also found a reduced risk of hip fracture among patients taking thiazide..$^{90}$ The only prospective study of the use of thiazide, however, was unable to show a persistent reduction in the rate of bone mineral loss. "Thus a causal relation between the use of thiazide and a reduced rate of bone loss has not yet been established.

The association between the use of thiazide and bone mass was first reported in a cohort of JapaneseAmerican men. ${ }^{2}$ We now report estimates of the effect of thiazide on rates of bone loss, based on a five year follow up of this cohort.

\section{Patients and methods}

We studied a cohort of Japanese-American men from a prospective epidemiological study of osteoporosis, which had started in 1981. The methods for identifying and recruiting the original cohort have been published previously. ${ }^{12}$ Briefly, a $30 \%$ random sample of surviving men from the Honolulu heart programme were invited to participate in a longitudinal epidemiological study of osteoporosis. These men were born between 1900 and 1920 and had been living on the island of Oahu at the first examination in 1981.
At the initial examination 1369 men had had measurements of bone mineral content at the following three sites: calcaneus (os calcis), distal radius, and proximal radius, using a rectilinear, single photon absorptiometric method. ${ }^{13}$ The calcaneus consists of roughly $95 \%$ trabecular bone with a thin cortical shell. The proximal radius site is predominantly cortical bone, and the distal radius site is roughly $40 \%$ trabecular and $60 \%$ cortical bone. The repeat measurements were obtained at roughly yearly intervals, during a mean follow up of five years. The mean number of measurement points was 4.0 for the radius sites and 4.9 for the calcaneus. The men ranged in age from 61 to 81 years, with a mean of $67 \cdot 8$ at the initial examination.

The smoking history, level of physical activity, presence of physical disabilities, history of chronic disease, and use of drugs were determined for each man. Height, weight, grip strength (dominant hand), and blood pressure were also measured.

Only men who had had two or more examinations were eligible for statistical analysis. Overall, 1329 men had had their bone mineral content measured at the calcaneus site and 1305 at the radius sites. Excluded from all analyses were men taking corticosteroids (17), oestrogens (5), or androgens (1); also excluded were two men who stopped taking thiazides within one year of the first examination. Reports of men taking thiazides that were not confirmed at two consecutive examinations led to the exclusion of 59 calcaneal and 78 radial measurements. Also, 179 men who were over the age of 75 at the first examination were excluded.

Individual rates of bone loss were calculated from regression of the bone mineral content measurements against age over years of subject observation or drug use. These loss rates were used as the dependent variable in multiple regression models including the use of thiazide and other covariates as the independent variables. All analyses were adjusted for height, weight, age, and whether the man smoked or not. Physical activity was not significant in these analyses, and adjusting for this did not influence the results. Similarly, mean bone mineral content was not significantly related to loss rate, and adjustment for mean bone mineral content did not substantially influence the results.

\section{Results}

Of the 1369 men, 325 had taken thiazide diuretics consistently on at least two consecutive examinations. The total duration of thiazide use could be established for $78 \%$ of the men; mean duration of use was 11.9 years. Hydrochlorothiazide was used most often, and the most common dose was $25 \mathrm{mg}$ daily. Rates of bone loss are shown in the table for the three measurement sites. The controls, who did not have hypertension, were compared with both the men taking thiazides and those taking other antihypertensive drugs. At all three sites the men taking other antihypertensive drugs had faster loss rates $(22 \cdot 6-43 \cdot 1 \%)$ than those of the controls but the difference was significant only for the distal radius. In contrast, the men taking thiazides had 


\begin{tabular}{|c|c|c|c|c|}
\hline Site & Treatment groups & $\begin{array}{l}\text { Mean rate of bone loss (units/year) } \\
(95 \% \text { confidence interval })\end{array}$ & $\begin{array}{l}\text { Rate of bone loss as } \% \text { of } \\
\text { control rate }\end{array}$ & $\mathrm{p}$ Value \\
\hline Calcaneus (mg/cm) & $\left\{\begin{array}{l}\text { Thiazide }(\mathrm{n}=325) \\
\text { Other drug }(\mathrm{n}=53) \\
\text { Control }(\mathrm{n}=639)\end{array}\right.$ & $\begin{array}{l}1 \cdot 50(0 \cdot 85 \text { to } 2 \cdot 16) \\
4 \cdot 22(2 \cdot 61 \text { to } 5 \cdot 83) \\
2 \cdot 95(2 \cdot 48 \text { to } 3 \cdot 41)\end{array}$ & $\begin{array}{l}-49 \cdot 2 \\
+43 \cdot 1\end{array}$ & $\begin{array}{l}0 \cdot 0005 \\
0 \cdot 14\end{array}$ \\
\hline Proximal radius $(\mathrm{g} / \mathrm{cm})$ & $\left\{\begin{array}{l}\text { Thiazide }(n=301) \\
\text { Other drug }(n=45) \\
\text { Control }(n=624)\end{array}\right.$ & $\begin{array}{l}0.00298(0.00140 \text { to } 0.00457) \\
0.00668(0.00263 \text { to } 0.01073) \\
0.00545(0.00436 \text { to } 0.00654)\end{array}$ & $\begin{array}{l}-45 \cdot 3 \\
+22 \cdot 6\end{array}$ & $\begin{array}{l}0.01 \\
0.57\end{array}$ \\
\hline Distal radius $(\mathrm{g} / \mathrm{cm})$ & $\left\{\begin{array}{l}\text { Thiazide }(n=300) \\
\text { Other drug }(n=45) \\
\text { Control }(n=624)\end{array}\right.$ & $\begin{array}{l}0.00703(0.00509 \text { to } 0.00897) \\
0.01258(0.00761 \text { to } 0.01754) \\
0.00988(0.00854 \text { to } 0.01122)\end{array}$ & $\begin{array}{l}-28 \cdot 8 \\
+27 \cdot 3\end{array}$ & $\begin{array}{l}0 \cdot 02 \\
0 \cdot 03\end{array}$ \\
\hline
\end{tabular}

^Figures normalised to $100 \%$.

†For difference compared with controls.

significantly slower loss rates at all three sites. The reductions in loss rate ranged from $28.8 \%$ (distal radius) to $49 \cdot 2 \%$ (calcaneus) relative to the controls No consistent effects of dose or duration were found.

\section{Discussion}

We have found significantly slower rates of bone loss at three skeletal measurement sites among men with hypertension taking thiazide diuretics. This result contrasts with that for the men taking antihypertensive drugs other than thiazides, who had faster loss rates than controls. Thus hypertension in itself does not appear to explain the slower rate of bone loss among men taking thiazides, which agrees with data from our previous, cross sectional study.

We are aware of only one previous, prospective study of thiazides and bone loss. In a two year study Transbol et al measured bone loss at the distal radius among 54 recently postmenopausal women with an average age of $50 .^{11}$ The women were randomised to take either $5 \mathrm{mg}$ bendrofluazide daily or a placebo; both groups, however, were given calcium supplements $500 \mathrm{mg} /$ day. Transbol et al found an apparent transient slowing of loss rate among women taking thiazides and concluded that thiazides were not an alternative to oestrogen replacement. Their study differed from ours in several respects. Their patients were newly postmenopausal women, in whom the effects of oestrogen deficiency may have been predominant. Also, both the control and treatment groups were given calcium supplements, which may also have influenced the rate of bone loss; therefore, part of the difference between the groups may have been obscured. Finally, the short duration of the study and small sample size would have made it difficult to show significant reductions in rates of bone loss of the magnitude found in our study.

Several cross sectional studies have found greater bone mass among patients taking thiazides. In this same cohort of men we have reported significantly higher bone mineral content among men with a mean duration of $7 \cdot 25$ years of thiazide use. ' Among the wives of these men we have also reported higher bone mineral content and reduced prevalence of fractures in those who were taking thiazides. ${ }^{3}$ Hale et al reported a lower prevalence of fractures among women taking thiazides. ${ }^{+}$Subsequently, there have been several reports of greater bone mass among women with hypertension taking thiazides. ${ }^{5-8}$

More recently, two studies have reported a reduced risk of hip fracture associated with the use of thiazides. Ray et al reported a case-control study based on computerised pharmacy records in the Canadian province of Saskatchewan. ${ }^{9}$ They found a significantly lower risk of hip fracture related to the duration of thiazide use for both men and women. For those patients taking thiazides for six or more years the relative risk of hip fracture was 0.5 compared with those not taking the drug. LaCroix et al have also reported a prospective study in three communities in the United States, in which the use of thiazide diuretics was associated with a one third reduction of the risk of hip fracture. ${ }^{10}$ Duration of thiazide use could not be established in this study.

Although these studies suggest a relation between the use of thiazides and improved skeletal strength, they cannot definitively determine whether a cause and effect relation exists. Thus some investigators have suggested the need for randomised, controlled studies. ${ }^{29}$

Our data provide more direct evidence of beneficial effect of thiazides on bone loss. The magnitude of the effect is interesting; the almost $50 \%$ reduction in loss rate at the trabecular calcaneal site seems to be greater than the reduction produced by calcium supplements in the same population, particularly for trabecular bone. ${ }^{1+}$ This observation raises questions about the potential mechanisms of thiazide's effect on bone. If the mechanism was solely related to improved calcium balance resulting from reduced urinary calcium we would expect the magnitude and distribution of the effect to be similar to that produced by calcium supplements.

There are data to suggest other possible mechanisms. Parathyroid hyperplasia has been reported in dogs given hydrochlorothiazide for several months. ${ }^{15}$ No increases in circulating parathyroid hormone levels, however, have been shown. ${ }^{16}$ Parfitt has suggested that the thiazide diuretics may potentiate the action of parathyroid hormone on both kidney and bone. ${ }^{18}$ In studies of absorptive hypercalciuria Pak et al reported that thiazides reduced urinary calcium excretion but that intestinal calcium absorption remained persistently raised..$^{19}$ It has also been suggested that the effect of thiazides on calcium metabolism depends on intact parathyroid glands..$^{20}$ Other known effects of thiazides include changes in concentrations of serum and urinary zinc, phosphate, and magnesium. The potential relations between these factors and bone loss are unknown.

Our data presented here provide additional evidence that thiazide diuretics in elderly patients can reduce rates of bone loss and thus the frequency of fractures. Further studies of the mechanism of this effect are needed. Randomised, controlled trials of thiazides, bone loss, and incidence of fracture are also warranted.

1 Dirks JH, Sutton RAL, eds. Diuretics: phvsiology, pharmacology and clinical use. Philadelphia: W B Saunders, 1985.

2 Wasnich RD, Benfante RJ, Yano K, et al. Thiazide effect on the mineral content of bone. $N$ Engl f Med 1983;309:344-7.

3 Wasnich RD, Ross PD, Heilbrun LK, et al. Differential effects of thiazide an estrogen upon bone mineral content and fracture prevalence. Obstet (ivnecol 1986;67:457-62.

4 Hale WE, Stewart RB, Marks RB. Central nervous system symptoms of elderly subjects using anti-hypertensive drugs. $\mathscr{f}$ Am Geriatr So $1984 \cdot 32 \cdot 5-10$.

5 lindsay R, Marshall B, Habouhi A, et al. Increased axial bone mass in women with hypertension: role of thiazide therapy [Abstract]. Fournal of Bone and Mineral Research 1987;2 (suppl 1):S29.

6 Sowers MR, Wallace RB, Lemke JH. Correlates of mid-radius bone densit among postmenopausal women: a community study. Am 7 Clin Nur 1985; 41:1045-53

Hunt I, Murphy N, Henderson C, et al. Bone mineral content in elderl women [Abstract]. Am f Epidemiol 1988; 128:903.

8 Caulev JA, Cummings SR. Thiazide diuretics preserve bone mass and reduce 
the risk of fractures in elderly women: a prospective study [Abstract]. fournal of Bone and Mineral Research 1989;4 (suppl):S171.

9 Ray WA, Downey W, Griffin MR, et al. Long-term use of thiazide diuretics and risk of hip fracture. Lancet 1989;i:687-90.

10 LaCroix AZ, Wienpahl J, White LR, et al. Thiazide diuretic agents and the incidence of hip fracture. $N$ Engl f Med 1990;322:286-90.

11 Transbol I, Christiansen MS, Jensen GF, et al. Thiazide for the postponement of postmenopausal bone loss. Metabolism 1982;31:383-6.
of

12 Heilbrun LK, Kagan A, Nomura A, Wasnich RD. The origins of epidemiologic studies of heart disease, cancer and osteoporosis among Hawaii Japanese. Hawaii Med f 1985;44:294-6.

13 Vogel JM, Cline JW, Harrison JF, et al. Microcomputer based dual energy photon absorptiometric bone mineral analyzer (VCH). IEEE Transaction on Nuclear Science 1979;26:576-82.

14 Johnson NE, Kutch R, Davis J, et al. The role of calcium in preventing bone loss [Abstract]. Fournal of Bone and Mineral Research 1989;4 (suppl 1):S175.
15 Pickleman J, Paloyan E, Forland M, et al. Thiazide induced parathyroid stimulation. Clin Res 1968;16:468.

16 Wilson DR, Honrath U, Sonnenberg H. Thiazide diuretic effect on medullary collecting duct function in the rat. Kidney Int 1983;23:711-6.

17 Woodhall PB, Tisher CC. Response of the distal tubule and cortical collecting duct to vasopressin in the rat. $\mathcal{F}$ Clin Invest 1973;52:3095-108.

18 Parfitt AM. Chlorothiazide-induced hypercalcaemia in juvenile osteoporosis and hyperparathyroidism. $N$ Engl f Med 1969;281:55-9.

19 Pak CYC, Nicar M, Northcutt C. The definition of the mechanism of hypercalciuria is necessary for the treatment of recurrent stone formers. Contrib Nephrol 1982;33:136-51.

20 Brickman AS, Massey SG, Coburn JW. Changes in serum and urinary calcium during treatment with hydrochlorothiazide: studies on mechanisms. f Clin Invest 1972;51:945-54.

(Accepted 20 September 1990)

\title{
Inappropriate use of laboratory services: long term combined approach to modify request patterns
}

\author{
D Bareford, A Hayling
}

Abstract

Objective-To see whether changes in request patterns for haematological tests could be influenced in the long term by information released from a haematology department.

Design-Analysis of request patterns by hospital divisions before and after intervention and of costs of intervention and savings achieved.

Setting-Haematology laboratory of an inner city district general hospital.

Interventions-Monthly release of a comparison of clinicians' workload statistics, issue of on call guidelines, and promulgation of information (by seminars and factsheets) on appropriate use of tests.

Main outcome measures-Request patterns before and after intervention.

Results-During the year after intervention requests fell by at least a fifth, and the reduction persisted over the next two years. The reduction was most pronounced in relation to inpatients within the division of medicine, for whom requests fell from an average of 4.0 per patient in the six months before intervention to 2.9 per patient in the six months after.

Conclusions - A definite and sustained reduction in inappropriate requests for laboratory investigations may be achieved by an ongoing policy of intervention including issuing guidelines and factsheets and holding seminars, but a positive attitude among senior consultant staff is crucial.

\section{Introduction}

There have been many attempts in the past decade to reduce the amount of inappropriate requests for tests received by hospital clinical laboratories (reviewed by Fraser and Woodford'). The apparent increase in inappropriate requests stems from several factors, ${ }^{23}$ including "routine" diagnostic testing ${ }^{4}$ fear of censure by seniors, ${ }^{5.7}$ entertainment of obscure diagnoses by junior medical staff, excessive frequency of repeat tests, ${ }^{8}$ and irrelevant test results stimulating further inappropriate testing. ${ }^{9}$ The gathering momentum of widespread inappropriate testing has been aided by the ever increasing automation of laboratory procedures, ${ }^{10}$ which make individual tests fairly cheap and the effort to control inappropriate requests comparatively expensive.

Attempts to control inappropriate requesting have included rationing tests, redesigning request forms, educating about appropriate tests for various conditions, educating about costs, issuing feedback information, and using protocols.' Many of these strategies failed completely, ${ }^{8112}$ and those that reduced requests were often expensive in time and manpower and had no sustained effect once they were withdrawn. ${ }^{13-16}$ This study was set up to see if a process of providing information and feedback to clinicians might be cost effective and retain long term influence.

\section{Methods}

All requests and "tests" (see below) received over the previous three and half years by the haematology department of an inner city district general hospital serving a catchment population of 262000 were analysed. A request was defined according to the Steering Group on Health Services Information's guidelines as a "pathology investigation on a single specimen or a group of related specimens taken from an individual patient and sent to a laboratory department at one time, regardless of the number of tests forming part of that investigation". ${ }^{17}$ A "test" was defined as a result or group of results obtained from one manipulation of a specimen. Thus several tests may derive from one request.

The laboratory receives requests from the accident and emergency department; in respect of inpatients and outpatients; from other, outlying hospitals; and from general practitioners. Requests from specific sources were analysed in more detail. Total requests by hospital divisions, split into inpatients and outpatients, were analysed on a monthly basis from February 1987 to December 1989 and compared with inpatient activity taken from deaths and discharges and outpatient activity taken from attendances using information from the patient administration system. On call requests were analysed for the divisions of medicine and surgery on a monthly basis from January 1987 to March 1990. Three tests (full blood count, erythrocyte sedimentation rate, and prothrombin time) carried out for the division of medicine were analysed on a monthly basis from September 1986 to January 1990.

Over eight months between October 1987 and July 1988 several interventions were initiated which might have led to more appropriate use of laboratory facilities. The interventions were costed and were as follows.

(1) From October 1987 each consultant received by post a monthly statement of usage of the laboratory by request and usage of three of the most commonly used tests showing his or her results compared with that of other clinicians subdivided by divisions. From July 1988 this was expanded to include the total cost of the request results provided to his or her clinical team.

(2) From February 1987, after agreement with clinical consultants, on call guidelines were issued to junior medical staff and brought to their attention 